\title{
Research on Coordination and Optimization of Conventional Bus Routes in Environment of Rail Transit
}

\author{
Xiaoju Zhao, a, Feigang Tan, b \\ ${ }^{1}$ Shenzhen Institute of Information Technology, Shenzhen, Guangdong, 518172, China \\ a zhaoxj@sziit.edu.cn, ${ }^{\mathrm{b}}$ tanfeigang@qq.com
}

\begin{abstract}
Keywords: Conventional Bus, Rail Transit, Coordination and Optimization, Integration of "Three Networks"

Abstract. We need to optimize and adjust the conventional bus network in the rail transit environment to give full play to the key role of the rail transit function and its overall benefits, to form smoothly linking combination of rail transit and conventional bus network. This paper is to analyze the defects of existing conventional bus network, and discuss the local optimization and adjustment of conventional bus routes in the attracting scope of rail transit in the case of the determination of rail network layout, and establish an optimization model of routes based on the maximum function of the delivery efficiency.
\end{abstract}

\section{Preface}

As the demand of urban transportation is unprecedentedly high, it has become an important step in the development of a fully functional public transport system to build the fast rail transportation system with a large capacity. With the continuous development of rail transit, as the differences of the technical and economic characteristics between rail transit and conventional bus, the construction of rail transit to conventional bus industry will make large impact or produce a variety of effects. To give full play to the key role of the rail transit and its overall benefits, it is necessary to adapt to the needs of different levels of passenger transport, which must be based on the coordination and integration between transportation ways. Recently, the idea of "three network integration of railway, bus, and slow walking to carry out the service supply-side reform" has clearly been put forward in some cities in China. The planning and coordination of rail transit and conventional bus network is the basis for constructing a reasonable public transport network and realizing the effective transfer in the networks, and it is of great significance to form the "three network integration" of railway network, bus network and slow walking network to build the integrated green public transportation system and enhance the appeal of public transport service.

\section{Content analysis of coordination and optimization}

For travelers, especially commuting traffic, there is a certain limit of walking time between the two ends no matter which form of public transportation the passengers use. According to a large number 
of surveys and statistical data analysis, $80 \%$ of passengers expect to control their walking time within 5-10 minutes between the two ends [1]. Of course, the passengers' acceptable walking time and waiting time are also related to the total time spent on the trip. Generally, the shorter the total time, the shorter the acceptable walking time, generally not exceeding $20 \%$ of the total time [2]. According to the mental and physical tolerance of passenger, the reasonable walking distance is no more than $600 \mathrm{~m}$. However, if we want to keep the walking distance for all the passengers of the rail transit within $600 \mathrm{~m}$, and without other feeder ways, the average density of rail transit network is at least $1.2 \sim 1.7 \mathrm{~km} / \mathrm{km} 2$, and it's hard to make it actually and is also not economic [3]. As a matter of fact, in terms of western cities where there is more mature development of rail transit, such as Paris, with the line layout of low load and high density, its network density is only $0.73 \mathrm{~km} / \mathrm{km} 2$, and the rail transit usually covers only a certain range of traffic corridors on both sides of the route because it is restricted by the network density [3]. Therefore, in order to take advantage of large capacity to ensure the traffic efficiency, Low-density rail networks must rely on bicycles, motorbikes, and especially conventional buses to provide secondary attraction of passengers, so the scope of service can be extended to all corners of the city to realize the rapid, safe, comfortable and orderly movement of passenger flow in urban space.

In China, the urban rail transit has not yet formed a perfect network. In order to ensure the formation of passenger corridors along rail transit and get a wide range of passenger flow, On the basis of the rail transit line, it is necessary to rationally match the fast and convenient gathering and distributing passenger flow of rail transit with conventional bus lines as large capacity, which complement and coordinate with each other so as to greatly improve the attraction of public transportation to form a reasonable urban public traffic network.

\section{Defects of conventional bus networks}

At present, there are main defects of conventional bus network in our country as follows:

(1) The distribution of bus lines can't reflect the needs of the distribution of passenger flow and the bus lines excessively concentrate on a few main roads, causing uneven distribution of public transport capacity, and some of bus lines on some roads is very crowded, there are no bus lines on some roads.

(2) The bus routes are too roundabout, as a result, the total bus travel distance of passengers is too long, and the operating cost of bus companies is also increased.

(3) Because it is unreasonable to set up the hub station on the bus network, the passengers' transferring times increase, and the distance is long.

(4) The parking area of the first and last stations of the bus network is insufficient and the bus approach rate is low, and some lines only turn around by the road, which affects road traffic.

The above defects are mainly caused by unreasonable bus network. As the bus network is the 
basis of the bus system, a perfect and reasonable public transport network must be established in order to give priority to the development of public transport.

\section{Principles of coordination and optimization}

The main theme of the coordination and optimization of rail transit and conventional bus line networks is that they should coordinate with each other, complement each other, without waste of resources caused by repeated construction, and pay attention to the relative balance of transportation facilities, we should follow the following principles for the combination design of the line network:

(1) Follow the path of the rail transit lines and combine the arrangement of the rail transit station to increase the conventional bus lines that are perpendicular to the rail line or in the direction of the intersection.

(2) Converge the starting point of the conventional bus lines on the ground at both ends of the fast rail transit line at the end of the rail transit line as far as possible, and add conventional bus line on the ground starting from the rail transit station to form a transferring station to pick up passengers of the rail transit and alleviate passenger flow fast and provide the convenience for passengers at the same time.

(3) Change the conventional bus lines on the ground and try to meet the rapid rail transit stations to facilitate interchange.

(4) For the rail transit line, we should retain some bus routes to play a role of certain diversion at the local section of the passenger flow oversaturation, but the overlap length should not exceed 4km, according to preliminary statistical analysis, it will lose the advantage of diversion if the overlap of the ground bus and rail transit exceeds more than $4 \mathrm{~km}$, [4].

In some prosperous areas of the city, the passenger flow is concentrated, which can't be fully assumed by the rail transit, so the conventional bus on the ground still plays a role of auxiliary diversion.

\section{Effect analysis of interaction between rail transit and conventional bus}

The effect type of interaction between rail transit and conventional bus can be divided into three kinds: effect of induced increase, effect of diversion and effect of complementarity. The effect of induced increase means when the rail transit is opened, it is more convenient and accessible to travel in the affected areas, which will induce the potential travel demand that can't travel or travel less frequently under the original traffic conditions. The effect of diversion refers to that the passenger flow of the original conventional bus is diverted to the rail transit because of the rapid, punctual advantages of rail transit with the opening of the rail transit. Under this circumstance that the layout of rail network is determined there is a competition area between conventional bus and rail transit, which needs to focus on the study of the repetition of conventional bus routes and rail 
transit lines. The effect of complementarity refers to that the citizens choose the appropriate way to travel and form complementarity according to the different characteristics of rail transit and conventional bus. At this point, the conventional bus routes have a cooperative relationship in addition to the competition within the scope of the attraction of rail transit, therefore, it is necessary to analyze the local optimization and adjustment of conventional bus, especially the analysis of the location of the bus station and the entrance and exit of the rail traffic, which is the key to realize "zero distance transfer" of the bus and subway and the integration of the three networks.

(1) The contention area between rail transit and conventional bus [5]

A large number of studies have shown that with the increase of travel distance, people are becoming more and more sensitive to travel time consumption. For long-distance travel, the travelers tend to choose the faster traffic way to shorten travel time. The time consumption of the conventional bus travel for residents once $T_{B}$ is shown in figure 1:

$$
T_{B}=\frac{D_{B} / 2}{V_{\text {步 }}}+\frac{T_{B \text { 间 }}}{2}+\frac{L-D_{B} / 2}{V_{B}}
$$

In the formula, the distance between the conventional bus stations is $500 \mathrm{~m}$;

$V_{\text {步 }}$ : the walking speed of $4.4 \mathrm{~km} / \mathrm{h}$;

$L$ : the travel distance of passengers; (ride distance, travel distance $=$ ride distance + walking distance at both ends)

$T_{B \text { 间 }}$ the time interval of conventional bus departure is $10 \mathrm{~min}$;

$V_{B}$ : the travel speed of conventional bus, the lower limit of $15 \mathrm{~km} / \mathrm{h}$, upper limit of $25 \mathrm{~km} / \mathrm{h}$;

The time consumption of rail transit for residents $T_{R}$, is shown in figure 2 :

$$
T_{R}=\frac{D_{R} / 2}{V_{\text {步 }}}+\frac{T_{R \text { 间 }}}{2}+\frac{L-D_{R} / 2}{V_{R}}
$$

Where,

$D_{R}$ : the distance of the rail transit stations of $1500 \mathrm{~m}$;

$V_{\text {步 }}$ : the walking speed of $4.4 \mathrm{~km} / \mathrm{h}$;

$T_{R \text { 间 }}$ : the time interval of rail transit departure is $6 \mathrm{~min}$;

$V_{R}$ : the travel speed of the rail transit, and the lower limit of $35 \mathrm{~km} / \mathrm{h}$ and the upper limit of $45 \mathrm{~km} / \mathrm{h}$;

According to the analysis of the two formulas, the advantages of rail transit are mainly manifested in long-distance travel, and there is no special advantages to travel less than $2 \mathrm{~km}$. The travel of rail transit and conventional bus is bound to form an interwoven zone, which is the main contention of rail transit and conventional bus, and it is about $2 \sim 7 \mathrm{~km}$ trip distance range of the residents [6]. 
(2) Attracting scope of rail transit

The attracting scope of rail transit includes primary attracting scope and secondary attracting scope, and the primary attracting scope, also called directly attracting, refers to the area of direct passenger flow attracted by rail transit, and it is the distribution range of passenger flow of walking to rail transit, and it is the reasonable pedestrian zone of rail transit. The secondary attracting scope, also called the indirect attraction scope, refers to the scope of the passenger flow area of the rail transit, which is the influence area of the rail transit.

Within the primary attracting scope of the rail transit, the competition between rail transit and conventional bus is a competitive relationship, therefore, within the primary attracting scope of the rail transit the conventional bus lines along the lines of the rail transit should be canceled. In the secondary attracting scope, the rail transit and the conventional bus belong to the relationship of mutual competition and cooperation. So during the layout of line network, we should coordinate the arrangement of rail transit network and conventional bus network in the secondary attracting scope of the rail transit, the conventional bus have the effect of delivering the passenger flow of rail transit, minimize the competition between rail transit and conventional bus, attract more passenger flow, and improve the efficiency of public transport.

\section{Optimization model of conventional bus routes [7]}

In this paper, the coordination of rail transit and the original conventional bus network is to adjust and optimize the conventional bus network in the attracting scope with the attracting scope of rail transit as the researching area and the rail transit line as the known route. For the coordination between the two, the general practice is to adjust conventional bus lines in combination with the actual situation on the basis of the four coordination principles mentioned above.

The advantages of rail transit attracting passenger flow are mainly reflected in that it is not affected by other traffic flow on the ground, and the speed is fast. Therefore, in order to optimize the local conventional bus network in the attracting scope of rail transit, we should consider two roles the bus network plays in the rail transit system:

(1) The conventional bus provides convenient and fast transfer for the on-and-off passengers flow of the rail transit system to meet the demand of traffic volume in the running of rail transit system;

(2) The conventional bus strengthens the function of rail transit system as a traffic way of medium-and-long distance. In this paper, we establish the model of line optimization with the maximum of the conventional bus delivery efficiency as the objective function. The efficiency of delivery refers to the ratio between the product of the number of passengers transported by the transferring bus route and the distance on the rail line (i.e. passenger turnover) and the length of the transferring bus routes. 
The calculation formula of delivery efficiency is as follows:

$$
\max E_{F}[i]=\frac{\sum_{i \in R, j \neq i}\left(q_{F R}(i, j)+q_{R F}(j, i)\right) \cdot l_{R}(i, j)}{l_{F}[i]}
$$

In the formula, $E_{F}[i]$ is the delivery efficiency of delivering route $\mathrm{F}$ originating from station $\mathrm{i}$ (person); $l_{R}(i, j)$ is the length $(\mathrm{km})$ from node $\mathrm{i}$ to node $\mathrm{j}$ on the rail route $\mathrm{R} ; l_{F}[i]$ is the length $(\mathrm{km})$ of delivering route $\mathrm{F}$ originating from station $\mathrm{i}$ (person); $q_{R F}(j, i)$ is the passenger flow (person) of rail route where the passengers get on at the station $\mathrm{j}$ and get off at the station $\mathrm{i}$ and then transfer to the delivering route $\mathrm{F} . q_{F R}(i, j)$ is the passenger flow (person) of rail route where the passengers get off the delivering route $F$ and get on at the station $i$ to the station $\mathrm{j}$.

$$
\begin{aligned}
& \left.q_{R F}(j, i)=\sum_{k=l}^{m} \max \overline{(f}[j, i], O D_{B}(j, k) \cdot \delta_{R F}(j, k)\right) \\
& q_{F R}(i, j)=\sum_{k=1}^{m} \max \left(\bar{f}[i, j], O D_{B}(k, j) \cdot \delta_{F R}(k, j)\right)
\end{aligned}
$$

In the formula, $O D_{B}(j, k), O D_{B}(k, j)$ is the number of OD (person) of the passenger demand of conventional bus between the station $j(k)$ of rail route and the station $k(j)$ of delivering route; $\bar{f}[i, j], \bar{f}[j, i]$ is the remaining passenger flow (enter) of the station $\mathrm{i}(\mathrm{j})$ to the station $\mathrm{j}(\mathrm{i})$; $\mathrm{m}$ is the destination of the delivering route (not transfer with diversion route); $\delta_{R F}(j, k), \delta_{F R}(k, j)$ is the orbit between the point $j(k)$ and $k(j)$ _ deliver(delivery__orbit) and transfer the competition parameters of travelling ways. If the short-circuit distribution method is used, there are:

$$
\begin{aligned}
& \delta_{R F}(j, k)=\left\{\begin{array}{l}
1, t_{R F}(j, k) \leq t_{B}(j, k) \\
0, t_{R F}(j, k) f t_{B}(j, k)
\end{array}\right. \\
& \delta_{R F}(j, k)=\left\{\begin{array}{l}
1, t_{F R}(k, j) \leq t_{B}(k, j) \\
0, t_{F R}(k, j) f t_{B}(k, j)
\end{array}\right.
\end{aligned}
$$

In the formula, $t_{R F}(j, k), t_{F R}(k, j)$ is the delivery time (hour) of the orbit between the point $j(k)$ and $k(j) \_$deliver(delivery__ orbit) and transfer travelling ways; $t_{B}(j, k), t_{B}(k, j)$ is the delivery time (hour) of competition mode (type) between the point $j(k)$ and $k(j)$;

The constraint conditions to be considered in the process of optimization are:

(1) The limitation of passenger flow capacity of rail routes, stations, bus routes and stations.

(2) The limitation of line length. It includes: the first is the length limitation of the feeder route. Generally speaking, the feeder bus line is about $6 \mathrm{~km}$ long, not more than $8 \mathrm{~km}$, and because the route is too long it complicates the function of the route. The second is the length limitation of bus lines parallel to rail transit. Its length should not exceed $4 \mathrm{~km}$, otherwise it will lose the advantage of diversion.

(3) Minimum bus operation input. It can be reflected by the maximum load rate or the minimum number of double track lines. 
Two important aspects should also be considered:

(1) The efficiency of the feeder route itself (route efficiency) and its effect on the rail line (the delivery benefit) should be large enough.

(2) The general route should avoid competing with the rail route, and the optimized search should be conducted outside the area of competition with the rail route.

\section{Conclusions}

In order to establish a reasonable hierarchical structure of urban public transport network, the first thing to solve is how to plan for a public transport network, and how to plan new rail transit lines and conventional bus lines to coordinate the alignment of rail transit lines and conventional bus lines, in order to build a reasonable urban public traffic line network with rail transit lines as the framework. In the construction of the network, it is necessary to consider the various factors involved, we can obtain a suitable and reasonable urban public transport network after analyzing and synthesizing the requirements and constraints of various aspects.

\section{Acknowledgement}

This work is funded by The Youth Training Project of school (QN201709).

\section{References}

[1] Levinson, H.S. Rail transit in the next millennium: Some global perspectives. Transiportation Research Record, 2000(in Chinese)

[2] Dure, Davis. Maximizing operating reliability in design of long single-track light rail transit lines. Transportation Reseach Record, 1999(in Chinese)

[3] Quan Yongji, Liu Xiaoming. Where is the way. China city press. 2002, 01(in Chinese)

[4] Jiang Fan. Research on connection between urban rail transit and other modes of transportation. Journal of Northern Jiaotong University, 2001.08(in Chinese)

[5] Wang Yuping. Competition and cooperation between conventional bus and rail transit: [master's thesis of Chang 'an university]. Xi 'an: Chang 'an university, 2004,1-79(in Chinese)

[6] White, P. (1995), Public Transport: its planning ,management and operation, Edition, UCL Press, 1995(in Chinese)

[7] Lu Huapu, zhu Jun, Wang Jianwei. Research and practice of urban rail transit planning. Beijing: China WaterPower Press, 2001,1-218(in Chinese) 